\title{
(2) OPEN ACCESS \\ Impact of the NHS Stop Smoking Services on smoking prevalence in England: a simulation modelling evaluation
}

\author{
Fujian Song $\odot,{ }^{1}$ Tim Elwell-Sutton, ${ }^{2}$ Felix Naughton ${ }^{3}$
}

\begin{abstract}
- Additional material is published online only. To view please visit the journal online (http://dx.doi.org/10.1136/ tobaccocontrol-2018-054879).
\end{abstract}

1 Norwich Medical School, University of East Anglia, Norwich, UK

${ }^{2}$ Public Health, Thurrock Council, Civic Offices, Essex, UK

${ }^{3}$ School of Health Sciences, University of East Anglia, Norwich, UK

\section{Correspondence to} Dr Fujian Song, Norwich Medical School, University of East Anglia, Norwich NR4 7TJ, UK; fujian.song@uea.ac.uk

Received 7 December 2018 Revised 31 January 2019 Accepted 9 February 2019 Published Online First 5 April 2019

\section{GLinked}

- http://dx.doi.org/10.1136/ tobaccocontrol-2018-054586. wit

Check for updates

(C) Author(s) (or their employer(s)) 2020. Re-use permitted under CC BY-NC. No commercial re-use. See rights and permissions. Published by BMJ.

To cite: Song F, Elwell-

Sutton T, Naughton $F$

Tob Control

2020:29:200-206.
ABSTRACT

Background The English National Health Service NHS Stop Smoking Services (SSS), established in 2001, were the first such services in the world. An appropriate evaluation of the SSS has national and international significance. This modelling study sought to evaluate the impact of the SSS on changes in smoking prevalence in England.

Methods A discrete time state-transition model was developed to simulate changes in smoking status among the adult population in England during 2001-2016. Input parameters were based on data from national statistics, population representative surveys and published literature. The main outcome was the percentage point reduction in smoking prevalence attributable to the SSS.

Results Smoking prevalence was reduced by $10.8 \%$ in absolute terms during 2001-2016 in England, and $15.3 \%$ of the reduction could be attributable to the SSS. The percentage point reduction in smoking prevalence each year was on average $0.72 \%$, and $0.11 \%$ could be attributable to the SSS. The proportion of SSS supported quit attempts increased from $5.5 \%$ in 2001, to as high as $18.9 \%$ in 2011, and then reduced to $8.2 \%$ in 2016 . Quit attempts with SSS support had a higher success rate than those without SSS support (15.1\% vs $11.3 \%$ ). Smoking prevalence in England continued to decline after the SSS was much reduced from 2013 onwards. Conclusions Approximately $15 \%$ of the percentage point reduction in smoking prevalence during 20012016 in England may be attributable to the NHS SSS, although uncertainty remains regarding the actual impact of the formal smoking cessation services.

\section{INTRODUCTION}

Cigarette smoking remains a leading cause of avoidable deaths worldwide. ${ }^{1}$ According to the WHO Framework Convention on Tobacco Control, comprehensive tobacco control measures include cigarette taxes, smoke-free laws, public information campaigns, advertising bans, health warning and cessation treatment. ${ }^{2}$ Observational evidence indicates that the implementation of key tobacco control measures is associated with increased cessation rates and reduced smoking prevalence at the population level. ${ }^{3}$ However, the relative impact of each of the key tobacco control measures on a population's smoking prevalence remains unclear, ${ }^{5}$ although different interventions, simultaneously implemented, may interact in complex ways and have synergistic effect.
The UK is considered to be in a leading position in Europe regarding the implementation of tobacco control measures. ${ }^{6}$ Smoking prevalence among adults in the UK has declined from $27 \%$ in 2000 to $16 \%$ in $2016 .^{7}$ As part of a comprehensive tobacco control strategy, National Health Service (NHS) Stop Smoking Services (SSS) were launched in Health Action Zones in 1999 in England, and were rolled out to all English Health Authorities in 2001. ${ }^{8}$ Smokers who are motivated to quit are referred by other health professionals or themselves to the SSS. The recommended total contact time for each client at SSS is 1.5 hours from pre-quit preparation to 4 weeks after quitting. ${ }^{9}$ Evidence-based behavioural and pharmacological interventions are provided by smoking cessation specialists, or delivered by staff in primary care, pharmacy or dental practice who have received smoking cessation training.

Previous studies have evaluated the effects of the SSS on short-term quitting ${ }^{8}$ and long-term success rates. ${ }^{10}{ }^{11}$ Other than a study using data from the SSS in one region in $2004,{ }^{12}$ the impact of the SSS on smoking prevalence in England has not been comprehensively quantified. As the first such services in the world, an appropriate evaluation of the SSS will provide empirical evidence for the development of tobacco control strategies in the UK and other countries. Based on official statistical data and published literature, we developed a computational simulation model to quantify the impact of the SSS on smoking prevalence between 2001 and 2016 in England.

\section{METHODS}

\section{Model framework}

A discrete time state-transition model ${ }^{13}$ was developed to simulate smoking prevalence among the adult population ( $\geq 16$ years old) in England between 2001 and 2016. The population was separated into subgroups by age, sex and smoking status. Smoking status was classified as never smokers, current smokers and former smokers. As the cycle length of time was 1 year in simulation modelling, current smokers in this study included those who quit smoking less than 12 months ago. Former smokers were defined as those who stopped smoking for at least 12 months and were further classified according to time since cessation. The simulation was started using the adult population structure of 2001. Population subgroups by age and gender were updated annually according to the probability of death and smoking status transition. 


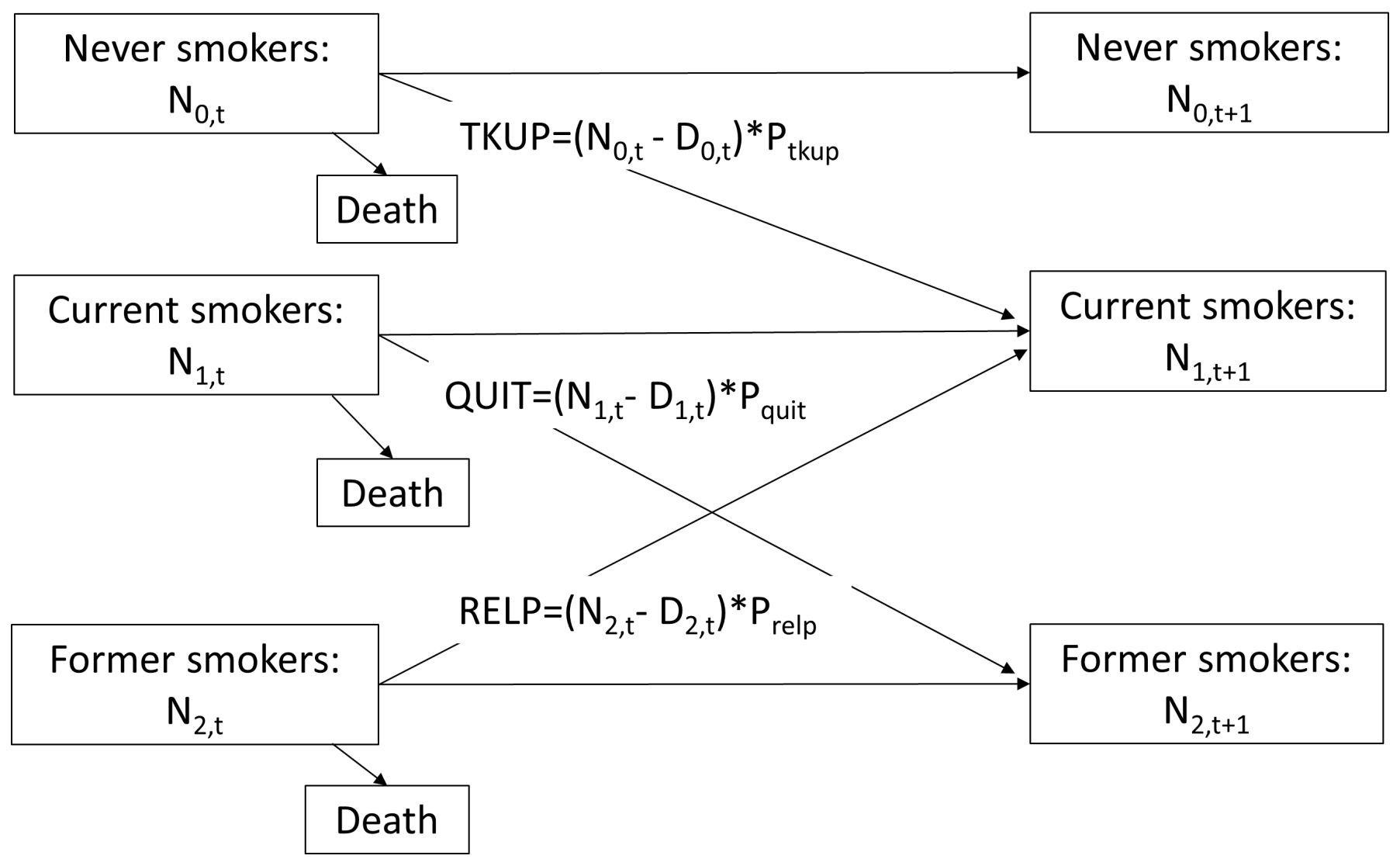

Figure 1 Diagram of possible transitions across smoking status. $D_{0, t^{\prime}} D_{1, t}$ and $D_{2, t^{\prime}}$ the number of deaths among never, current and former smokers in year $T_{;} N_{0, t^{\prime}} N_{1, t}$ and $N_{2, t^{\prime}}$ the number of never, current and former smokers in year $t$, respectively; $P_{\text {tkup, }}$ the probability of taking up smoking in never smokers; $P_{\text {quit' }}$ the probability of quitting for current smokers; $P_{\text {relp' }}$ the probability of returning to smoking among former smokers; $Q$ QUIT, the number of quitters; RELP, the number of former smokers who start to smoke again; TKUP, the number of new take-ups.

Detailed methods for the simulation modelling are provided in online supplementary appendix 1 . The transition between smoking status over time was based on (1) the probability of current smokers who quit smoking, (2) the probability of former smokers who start to smoke regularly again and (3) the probability of never smokers who start to smoke regularly. Figure 1 shows the possible transitions across never, current and former smokers between year (ie, at the beginning of the year) and year $t+1$. Never smokers in year $t$ may remain as never smokers or may start smoking. The number of never smokers in year $t+1$ equals the number of never smokers in year $t$ minus deaths and new take-ups. Similarly, current smokers in year $t$ may remain as current smokers or may have stopped smoking by the beginning of year $t+1$. The number of current smokers in year $t+1$ equals the number of current smokers in year $t$ minus deaths and quitters, plus new take-ups in year t. Finally, the number of former smokers at the beginning of year $t+1$ is calculated by subtracting deaths and relapsers from, and adding new quitters to, the number of former smokers in year t. Exogenous inputs to the model included the number of people aged 16 and net immigrants, along with their smoking status.

\section{Data sources and parameter estimation}

The 2001 mid-year population and death rates by age and sex in England were obtained from the Office for National Statistics. ${ }^{14}$
To estimate the size of the population at the start of the year 2001, we subtracted half of the difference between population estimates for 2000 and 2001 from the mid-year population in 2001. The prevalence rates for never, current and former smokers were based on data from General Lifestyle Surveys in the UK. ${ }^{715}$ Smoking prevalence was gender-specific and age-specific. Some of the fluctuation in the reported smoking prevalence rates during 2001-2016 was probably as a result of sampling errors. To facilitate simulation modelling, the observed age-specific and sex-specific smoking prevalence rates over time were smoothed by simple linear regression (online supplementary file 1).

Relative risks for death by smoking status were based on data summarised by Vugrin et al. ${ }^{16}$ New smoking take-ups included 16-year-old smokers, which were exogenously added to the simulated population. Then, we estimated the additional number of new take-ups among people aged 16-22 years in line with the observed increase in current and former smokers between two adjacent years (for details, see online supplementary file 1). The model allowed for relapse, with rates declining with the number of years quit, and it was assumed that relapse rates among those who quit for $\geq 12$ months were not affected by the SSS. The risk of smoking relapse for former smokers was estimated according to the relapse curve developed by Stapleton and West. ${ }^{17}$ The rate of quit attempts among current smokers was estimated using data from a series of Opinion Surveys in England ${ }^{18}$ and 


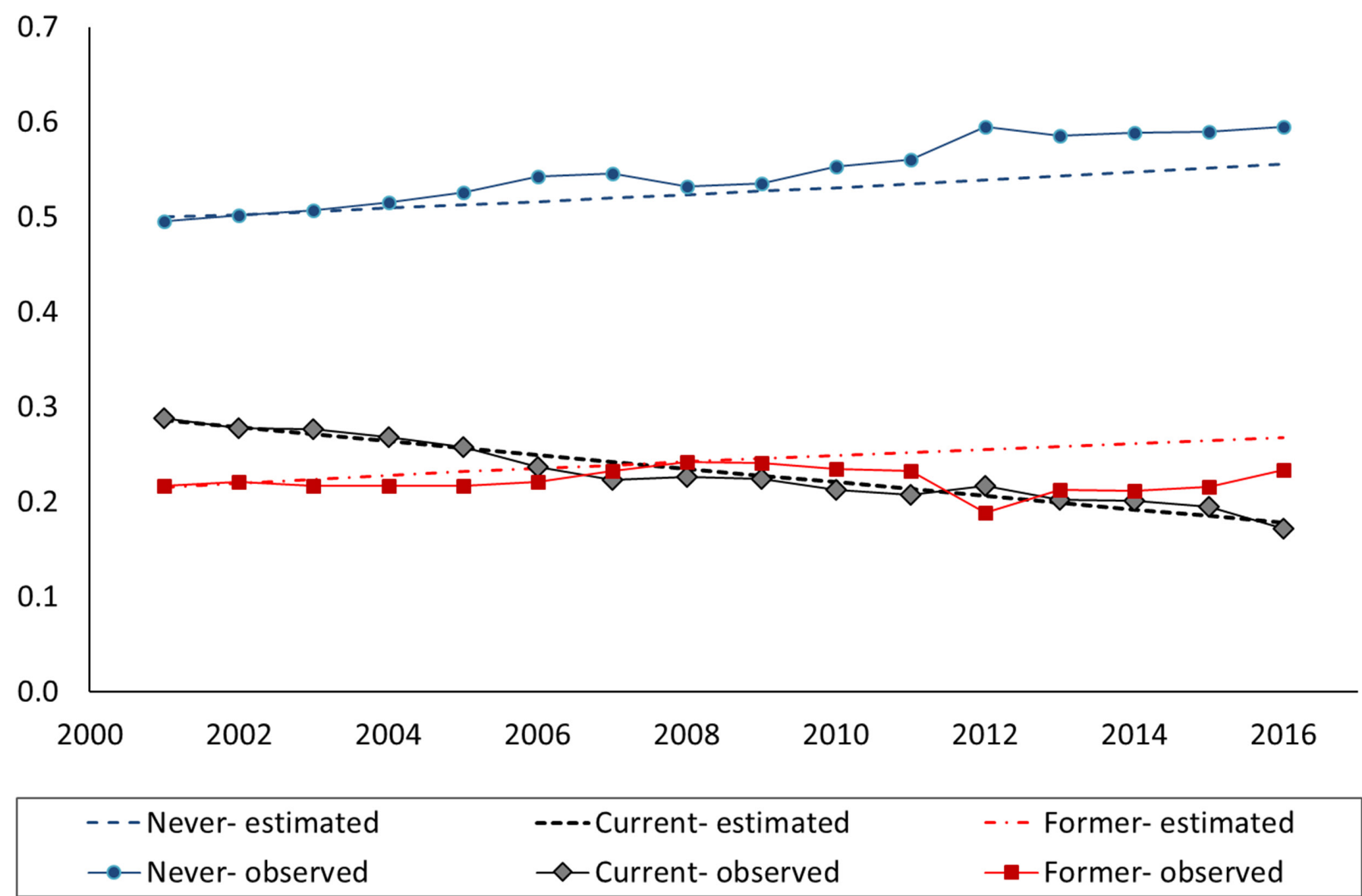

Figure 2 Prevalence rates of never, current and former smokers in England: the observed and estimated.

the Smoking Toolkit Study in England. ${ }^{19}$ The number of quit attempts supported by SSS and short-term quitters at 4 weeks after the quit date were based on SSS Statistics. ${ }^{20}$ The risk of smoking relapse by 12 months among short-term quitters was estimated according to data from three long-term follow-up studies, ${ }^{101121}$ and data from the Smoking Toolkit Study. ${ }^{22}$ To be consistent with the self-reported smoking and cessation status in national surveys in England, ${ }^{18}$ we used self-reported quit rates in the present study.

\section{Outcomes and data analyses}

The model's outcomes include the number of current smokers who attempted to quit smoking and the number of smokers who successfully quit each year from 2001 to 2016 in England. The quit attempts and successful quitters were further separated according to whether support from the SSS was received or not. Due to the limited availability of relevant data, the main outcomes were estimated by a novel modelling approach: the number of quitters in year $t$ was calculated based on the change in smoking prevalence between year $\mathrm{t}$ and $\mathrm{t}+1$, given estimated smoking relapse, new take-ups and death (see online supplementary appendix 1: figure 2). We first calculated the number of current smokers at the beginning of a year by assuming there were no smokers who stopped smoking in the past 12 months, given other inflow to and outflow from the pool of current smokers. Then we calculated the number of current smokers based on the observed smoking prevalence. The difference in the number of current smokers between the two calculations was used to estimate the number of all quitters required in the past year to achieve the observed smoking prevalence in the current year. The number of quitters without SSS support was estimated by subtracting the estimated number of quitters with SSS support from the estimated number of all quitters (online supplementary appendix 1). In addition to the rates of current smokers, we also calibrated the relevant inflow and outflow parameters by comparing the estimated and observed proportions of never and former smokers in England.

Reduction in smoking prevalence over time is presented using percentage point change which is the arithmetic difference of two smoking prevalence rates in different years. We estimate the percentage point reduction in smoking prevalence attributable to the SSS, based on the total percentage point reduction and the proportion of quitters who received support from the SSS. Due to important changes in the SSS since 2013, including declining client numbers, ${ }^{23}$ public health budget cuts ${ }^{23} 24$ and increased use of e-cigarettes, ${ }^{25}{ }^{26}$ results of the evaluation were also considered separately for the period 2001-2012 and 20 013-2016.

The computing language $\mathrm{R}$ was used to perform the simulation modelling.

\section{RESULTS}

\section{Smoking status between 2001 and 2016}

Figure 2 shows the observed and estimated overall prevalence of current, former and never smokers in the population aged $\geq 16$ in England during 2001-2016. As the number of quitters was estimated based on changes in smoking prevalence over time, the estimated prevalence rates for current smokers agree closely with the reported prevalence rates. ${ }^{715}$ The overall rate of smoking prevalence (including those who quit smoking for $<12$ months) 


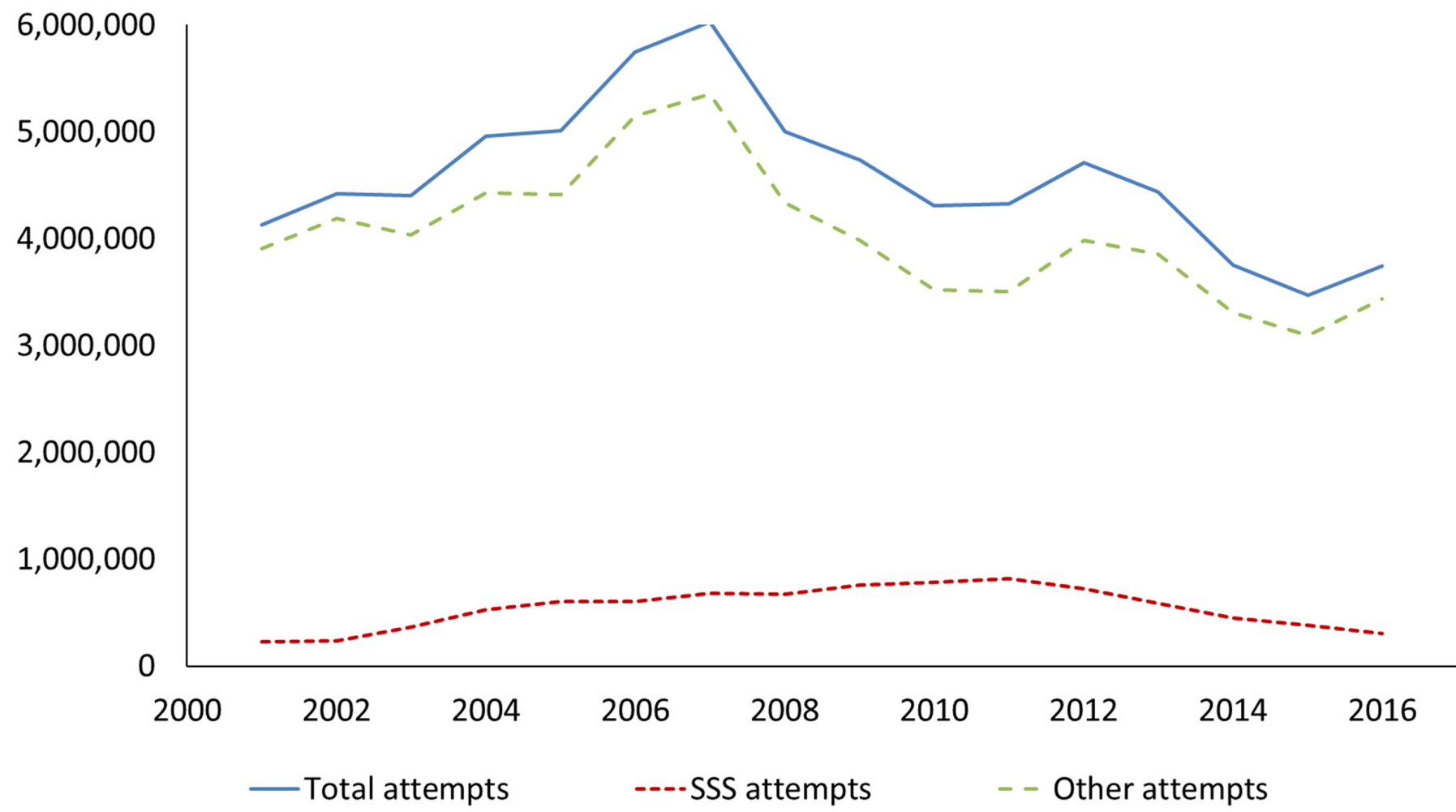

Figure 3 Estimated number of quit attempts in England between 2001 and 2016. The estimated number included multiple quit attempts by individual smokers within a year. Other attempts, quit attempts without support from Stop Smoking Services; SSS, quit attempts with support from Stop Smoking Services.

was estimated to be $28.6 \%$ in $2001,20.6 \%$ in 2012 and $17.8 \%$ in 2016. Compared with the observed rates in 2016, the estimated prevalence of never smokers in 2016 was lower $55.5 \%$ vs $59.5 \%$ ) and the estimated rates of former smokers was higher (26.7\% vs $23.3 \%)$. The differences between the estimated and observed rates of never and former smokers were likely to be a result of reclassification of former smokers ${ }^{727}$ which is considered in the discussion section.

The estimated number of current smokers (including those who quit smoking for $<12$ months) was reduced by $29.4 \%$ during 2001-2016, from 11.30 million in 2001 to 7.98 million in 2016. The absolute number of current smokers in England was on average reduced by approximately 211900 each year during 2001-2012, and by 239100 each year during 20132016 (online supplementary appendix 2).

The estimated number of new take-ups to smoking each year was reduced by $37.8 \%$ during 2001-2016, from 257300 in 2001 to 160100 in 2016 (online supplementary appendix 2). However, the number of relapsed smokers increased by $27.1 \%$, from 132800 in 2001 to 168800 in 2016, corresponding to an increase in the number of former smokers (from 8.5 million in 2001 to 12.0 million in 2016). Net immigration added around 50000 current smokers per year. The estimated number of the outflow from the population of current smokers was on average 642300 each year. The proportion of people contributing to the total outflow due to smoking cessation increased from $80.3 \%$ in 2001 to $86.1 \%$ in 2016 .

\section{Quit attempts and success between 2001 and 2016}

The estimated numbers of quit attempts in England during 2001-2016 are shown in figure 3 (and online supplementary appendix 3-4). On average, there were approximately 4.58 million quit attempts annually in England during 2001-2016.
The number of quit attempts by current smokers peaked at 6.03 million (60.0\% of all current smokers) in 2007. On average, around 545000 smokers set a quit date at SSS per year during 2001-2016, and this accounted for $11.9 \%$ of all quit attempts. The number of quit attempts at SSS peaked at around 816400 in 2011 , in contrast to a decline in the total number of quit attempts in England during 2007-2011 (figure 3). The proportion of SSS supported quit attempts increased from $5.5 \%$ in 2001 , to as high as $18.9 \%$ in 2011 , and then reduced to $8.2 \%$ in 2016.

The estimated number of long-term quitters in England was on average 538400 each year during 2001-2016, and 15.3\% of the long-term quitters received support from the SSS (online supplementary appendix 3). Quit attempts with SSS support had a higher success rate, compared with other quit attempts $(15.1 \%$ vs $11.3 \%)$.

The estimated proportion of SSS supported quitters (15.3\%) was linked with the assumed probability of smoking relapse and take-ups. Therefore, we conducted some post-hoc sensitivity analyses by increasing or lowering the probability of relapse and take-ups. If the risk of relapse and take-ups was both increased by $20 \%$ (consequently a larger number of quitters would be required to achieve the observed reduction in smoking prevalence), the estimated proportion of SSS supported quitters would be reduced to $12.3 \%$. If the risk of relapse and take-ups was both reduced by $20 \%$, the proportion of SSS supported quitters was increased to $17.5 \%$ during 2001-2016.

The prevalence rate for current smokers in England was reduced by $10.8 \%$ in absolute terms, from $28.6 \%$ in 2001 to $17.8 \%$ in 2016. We estimate, therefore, that $1.65 \%$ (ie, $10.8 \times 0.153$ ) of the reduction in smoking prevalence during 2001-2016 was attributable to the SSS (table 1). The average percentage point reduction in smoking prevalence was $0.72 \%$ (ie, $10.8 \% / 15$ ) each 
Table 1 Estimated impact of the Stop Smoking Services (SSS) on the reduction in smoking prevalence in England: 2001-2016

\begin{tabular}{|c|c|c|c|c|}
\hline Year & Percentage point reduction in smoking prevalence & $\begin{array}{l}\text { Annual percentage point } \\
\text { reduction in smoking prevalence }\end{array}$ & $\begin{array}{l}\text { Average proportion of SSS } \\
\text { supported quitters }\end{array}$ & $\begin{array}{l}\text { Annual prevalence } \\
\text { reduction attributable to } \\
\text { SSS }\end{array}$ \\
\hline 2001-2012 & $8.0 \%$ (from $28.6 \%$ in 2001 to $20.6 \%$ in 2012 ) & $0.727 \%$ & $15.68 \%$ & $0.114 \%$ \\
\hline 2012-2016 & $2.8 \%$ (from $20.6 \%$ in 2012 to $17.8 \%$ in 2016 ) & $0.700 \%$ & $14.03 \%$ & $0.098 \%$ \\
\hline 2001-2016 & $10.8 \%$ (from $28.6 \%$ in 2001 to $17.8 \%$ in 2016 ) & $0.720 \%$ & $15.26 \%$ & $0.110 \%$ \\
\hline
\end{tabular}

year from 2001 to 2016, and $0.11 \%$ percentage point reduction each year could be attributable to support from the SSS.

\section{DISCUSSION}

As the first such services established anywhere in the world, an appropriate evaluation of the SSS provides important information for the development of public health policies regarding tobacco control in the UK and other countries. In the present study, we developed a computational model based on data from a variety of sources to evaluate the impact of the SSS on reductions in smoking prevalence in England. Our estimated percentage point reduction in smoking prevalence attributable to SSS support was $0.11 \%$ each year during 2001-2016, which was near the lower boundary of a previous estimate $(0.1 \%-0.3 \%)$ using SSS data from one region in England during 2003-2004. ${ }^{12}$ Another study estimated that a third of smoking prevalence reduction $(0.24 \%$ out of $0.79 \%$ ) in 2014 in England could be attributable to cessation aids. ${ }^{28}$ In addition, our estimated annual percentage point reduction $(0.11 \%)$ attributable to the SSS was somewhat smaller than the annual percentage point reduction of $0.157 \%$ estimated by Gravely et $a l^{3}$ for implementing cessation support at the highest level in a population. However, the two estimates seemed broadly consistent in scale, considering that our estimated percentage point reduction was attributable to the SSS only, and cessation treatments are also available outside the SSS.

The main difference in cessation support between SSS and non-SSS quit attempts was the provision of varenicline (only available on prescription) and behavioural counselling. ${ }^{8}$ According to the results of the present study, the overall rates of success during 2001-2016 were higher for quit attempts with SSS support, compared with those without such support $(15.1 \%$ vs $11.3 \%)$. This result is consistent with findings from previous studies. One study estimated that biochemically verified abstinence at 4 weeks was about $35 \%$ with SSS support, and $25 \%$ for those with only a prescription of smoking cessation medication. ${ }^{8}$ Within the SSS, cessation support may be provided by dedicated cessation specialists, or by trained general practitioners, nurses, pharmacists or dentists. ${ }^{29}$ Evidence shows that the rate of relapse at 12 months is lower among short-term quitters who received specialist support compared with those receiving non-specialist support. $^{30}$

The impact of cessation support will depend on how many current smokers initiate a quit attempt. The proportion of quit attempts among current smokers was highest in 2006/2007 which may be explained by the ban on smoking in enclosed public places and workplaces after the introduction of smokefree legislation in July 2007 in England. ${ }^{31}$ The proportion of quit attempts with SSS support increased from 5.5\% in 2001 to as high as $18.9 \%$ in 2011 . However, the total number of quit attempts in England did not correspondingly increase, indicating a simultaneous reduction in quit attempts without SSS support (figure 3). Of all long-term quitters, the proportion of quitters who received support from the SSS reduced from 15.7\% during
2001-2012 to $14.0 \%$ during 2013-2016. Using data from statistics on smoking in England, ${ }^{7}$ figure 4 shows the changes in smoking prevalence and the implementation of several tobacco control measures in the UK during 1990-2016. Smoking prevalence was reduced by $0.30 \%$ per year during $1990-2000$ and reduced by $0.85 \%$ per year during $2000-2007$. The decline during 2000-2007 was at least partially attributable to both the SSS and the ban on smoking in public places in 2007, although other relevant tobacco control activities will also have contributed to this (see figure 4). ${ }^{31}$ However, the reduction in smoking prevalence slowed during 2007-2012, to only $0.10 \%$ per year, even with an increased number of SSS users. Then, the decline in smoking prevalence increased to $1.08 \%$ per year during 2012 2016 , in a period when the use of the SSS was much reduced. The recent faster reduction in smoking prevalence may be due to the increased use of electronic cigarettes and other tobacco control measures. ${ }^{2526}$

In summary, we estimate that approximately $15 \%$ of the reduction in smoking prevalence during 2001-2016 in England was associated with the SSS. However, the actual impact of the SSS is likely to be smaller than this because some of the longterm quitters with SSS support, it may be assumed, would have quit smoking anyway even without SSS support. In addition, the provision of cessation support has a limited effect on the initiation of quit attempts, as revealed by the fact that an increased number of quit attempts with SSS support was accompanied with a reduced total number of quit attempts during 2007-2011.

Since 2013, the SSS has changed due to both declining client numbers $^{23}$ and public health budget cuts, ${ }^{24}$ with much less support available from specialist smoking cessation advisors. Although specialist cessation support may not be necessary to all quit attempts, it may improve quit success for some. It has been advocated that the English SSS should be a mix of specialist support for a small number of smokers who really want it, brief support plus cessation medication for many and self-help for the majority. ${ }^{32}$ Future studies, including modelling evaluations, are required to compare different strategies of SSS and other tobacco control measures.

\section{Strengths and Limitations}

The impact of the SSS on smoking prevalence cannot be assessed in randomised controlled studies, and so a modelling approach is required. There have been several previous simulation modelling studies on smoking prevalence or cost-effectiveness of smoking cessation services in the UK. ${ }^{6} 2733{ }^{34}$ However, in previous modelling studies, the impact of cessation programmes on smoking prevalence was always considered as a known parameter, rather than an outcome of the evaluation. For example, a modelling study on smoking prevalence was based on the assumption that cessation treatment would reduce smoking prevalence by $4.75 \%$ during $1998-2010$ in the UK. ${ }^{6}$ Using realworld data and a novel modelling approach, the present modelling study is the first to assess the impact of smoking cessation 


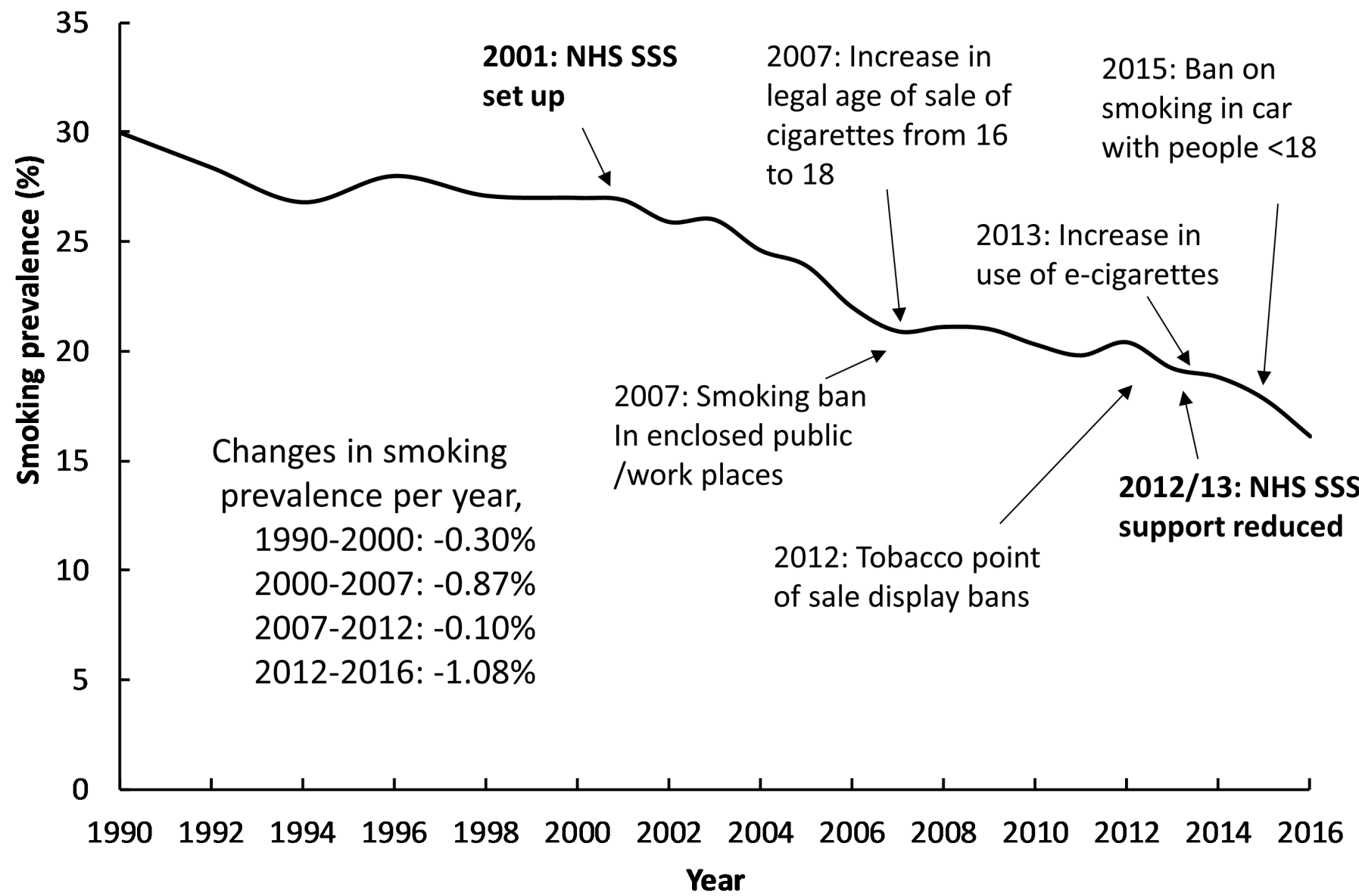

Figure 4 Changes in smoking prevalence and implementation of tobacco control measures in England. Smoking prevalence originally reported in official statistics. NHS, National Health Service; SSS, Stop Smoking Services.

services on smoking prevalence over time, by considering such impact as an outcome of the evaluation.

One specific feature of the present simulation modelling was to calibrate the number of smokers who stopped smoking according to the change in smoking prevalence. As expected, the estimated rates of smoking prevalence in England during 2001-2016 were similar to the observed smoking prevalence. However, the estimated rates of never smokers were lower, and the estimated rates of former smokers were higher, compared with the reported rates based on survey studies (figure 2). These differences have been reported previously as the phenomenon of 'reclassification'. ${ }^{27}$ That is, former smokers might consider themselves as never smokers after having quit for many years. In addition, a change in the definition of never smokers for people aged 16 and 17 led to an increase in the reported proportion of people who were never smokers in England since 2012 (from $56.0 \%$ in 2011 to about $59.5 \%$ in $2012-2016$ ), and simultaneously a reduction in rates of former smokers. ${ }^{7}$

The number of quit attempts and short-term quitters in the SSS was relatively certain because of availability of official statistics for these services. However, the number of 12 month quitters was estimated based on several studies of long-term outcomes among SSS clients. For the total number of quit attempts, we used data from a series of national surveys in England. ${ }^{18} 19$ It is unclear whether the observed fluctuations in the reported rates of quit attempts were true or due to sampling errors. The rates of smoking relapse, relative risk of death for never, current and former smokers are all estimated based on limited literature or datasets. Due to a lack of data from studies in England, we used data from the USA on relative risks of death for former smokers by time since cessation. For simplicity, we used a deterministic modelling approach without explicitly incorporating uncertainty and sampling errors, although the results on average are unlikely to be different after incorporating sampling errors into the simulation modelling.

There are further limitations in the present study, including that we did not evaluate the cost-effectiveness of the SSS for smoking cessation. Smokers who quit smoking using SSS support may be systematically different from those who quit without SSS support. The current study focused on the overall smoking prevalence by age and gender, without considering differences in smoking prevalence by socioeconomic status. In addition, we focused on the impact of the SSS, and did not explicitly consider cessation support from other sources, including the use of e-cigarettes. The present study focused on the SSS in England, and results may not be generalisable to other nations (Scotland, Wales and North Ireland) in the UK. Finally, available data were insufficient to estimate how many quitters with SSS support would have quit smoking anyway even without SSS support. However, the current study provides unique empirical evidence regarding the impact of smoking cessation support delivered through a national network of formal cessation services on prevalence.

\section{CONCLUSIONS}

Approximately $15 \%$ of the percentage point reduction in smoking prevalence during 2001-2016 may be attributable to the NHS SSS in England, although the actual impact of the 
formal smoking cessation services remains uncertain as some people who stopped smoking with support from the SSS would have quit anyway without formal and intensive support.

\section{What this paper adds}

\section{What is already known on this subject?}

- The implementation of key tobacco control measures, including cigarette taxes, smoke-free laws, public information campaigns, advertising bans, health warning and cessation treatment, is associated with increased cessation rates and reduced smoking prevalence at the population level.

What important gaps in knowledge exist on this topic?

- The relative impact of each of the key tobacco control measures on a population's smoking prevalence remains unclear.

- As the first such services in the world, the impact of the National Health Service Stop Smoking Services (SSS) on smoking prevalence in England has not been comprehensively quantified.

\section{What this paper adds?}

- Based on official statistical data and published literature, we developed a novel computational model to quantify the impact of the SSS on smoking prevalence between 2001 and 2016 in England.

- Smoking prevalence was reduced by $10.8 \%$ in absolute terms between 2001 and 2016 in England, and approximately 15\% of this reduction could be attributable to the SSS.

\section{Acknowledgements We thank Professor Robert West for providing helpful} comments on the draft manuscript.

Contributors FS conceptualised the initial idea, searched literature, collected data, developed the simulation model and prepared the draft manuscript. TE-S and FN critically commented on draft manuscripts and helped interpret modelling results. FS has access to the data and accepts full responsibility for the work.

Funding The authors have not declared a specific grant for this research from any funding agency in the public, commercial or not-for-profit sectors.

Competing interests None declared.

Patient consent for publication Not required.

Provenance and peer review Not commissioned; externally peer reviewed.

Open access This is an open access article distributed in accordance with the Creative Commons Attribution Non Commercial (CC BY-NC 4.0) license, which permits others to distribute, remix, adapt, build upon this work non-commercially, and license their derivative works on different terms, provided the original work is properly cited, appropriate credit is given, any changes made indicated, and the use is non-commercial. See: http://creativecommons.org/licenses/by-nc/4.0/.

\section{ORCID iD}

Fujian Song http://orcid.org/0000-0002-4039-1531

\section{REFERENCES}

1 GBD 2015 Tobacco Collaborators. Smoking prevalence and attributable disease burden in 195 countries and territories, 1990-2015: a systematic analysis from the global burden of Disease Study 2015. Lancet 2017;389:1885-906.

2 WHO. Who framework convention on tobacco control, 2003. Available: http://www. who.int/fctc/about/en/ [Accessed 14 May 2018]

3 Gravely S, Giovino GA, Craig L, et al. Implementation of key demand-reduction measures of the WHO framework convention on tobacco control and change in smoking prevalence in 126 countries: an association study. Lancet Public Health 2017;2:e166-74.

4 Feliu A, Filippidis FT, Joossens L, et al. Impact of tobacco control policies on smoking prevalence and quit ratios in 27 European Union countries from 2006 to 2014. Tob Control 2019;28:101-9.
5 Warner KE. Understanding the association between the WHO framework convention on tobacco control, adoption of tobacco control policies, and reduction in smoking prevalence. Lancet Public Health 2017;2:e158-9.

6 Levy DT, Currie L, Clancy L. Tobacco control policy in the UK: blueprint for the rest of Europe? Eur J Public Health 2013;23:201-6.

7 HSCIC. Statistics on Smoking England 2017: Health \& Social Care Information Centre, 2017.

8 West R, May S, West M, et al. Performance of English stop smoking services in first 10 years: analysis of service monitoring data. BMJ 2013;347:f4921.

9 DoH. NHS stop smoking services: service and monitoring guidance 2010/11. London: Department of Health, 2009.

10 Dobbie F, Hiscock R, Leonardi-Bee J, et al. Evaluating long-term outcomes of NHS stop smoking services (ELONS): a prospective cohort study. Health Technol Assess 2015;19:1-156.

11 Ferguson J, Bauld L, Chesterman J, et al. The English smoking treatment services: oneyear outcomes. Addiction 2005;100 Suppl 2:59-69.

12 Milne E. NHS smoking cessation services and smoking prevalence: observational study. BMJ 2005;330.

13 Mendez D, Warner KE, Courant PN. Has smoking cessation ceased? expected trends in the prevalence of smoking in the United States. Am J Epidemiol 1998;148:249-58.

14 ONS. User requested data: English population estimates and deaths by sex and single year of age, 1993 to 2013, 2016. Available: https://www.ons.gov.uk/ [Accessed 1 Feb 2017].

15 ONS. General lifestyle survey overview. A report on the 2010 General lifestyle survey. In: Office for national statistics, 2012.

16 Vugrin ED, Rostron BL, Verzi SJ, et al. Modeling the potential effects of new tobacco products and policies: a dynamic population model for multiple product use and harm. PLoS One 2015:10:e0121008.

17 Stapleton JA, West R. A direct method and ICER tables for the estimation of the cost-effectiveness of smoking cessation interventions in general populations: application to a new cytisine trial and other examples. Nicotine Tob Res 2012;14:463-71

18 ONS. Opinion survey report no. 40. smoking-related behaviour and attitudes, 2008/09. Newport, South Wales: NHS Information Centre for health and social care. Office for National Statistics, 2009.

19 West R, Brown J. Latest trends on smoking in England from the smoking toolkit study (updated: 5th September 2017). Available: http://www.smokinginengland.info/stsdocuments/ [Accessed 20 Mar 2018].

20 HSCIC. Statistics on NHS stop smoking services England, 2017. The Health and Social Care Information Centre, 2017.

21 Blyth A, Maskrey V, Notley C, et al. Effectiveness and economic evaluation of self-help educational materials for the prevention of smoking relapse: randomised controlled trial. Health Technol Assess 2015;19:1-70.

22 Brown J, West R. Brief report: quit success rates in England 2007-2017. In: Smoking in Britain. 2017: 5, 1-8.

23 Mayor $\mathrm{S}$. Fewer people are quitting smoking, say NHS stop smoking services. BMJ 2016;354.

24 Wise J. Stop smoking services are under threat owing to budget cuts. BMJ 2016;352.

25 Beard E, West R, Michie $S$, et al. Association between electronic cigarette use and changes in quit attempts, success of quit attempts, use of smoking cessation pharmacotherapy, and use of stop smoking services in England: time series analysis of population trends. BMJ 2016;354.

26 Britton J. Electronic cigarettes and smoking cessation in England. BMJ 2016;354.

$27 \mathrm{Kemm}$ J. A model to predict the results of changes in smoking behaviour on smoking prevalence. J Public Health 2003;25:318-24.

28 West R, Brown J. "The Smoking Pipe": A model of the annual inflow and outflow of cigarette smokers in England in 2014. In: Smoking in Britain. , 2015: 3, 1-9.

29 Csikar Jl, Douglas GV, Pavitt S, et al. The cost-effectiveness of smoking cessation services provided by general dental practice, general medical practice, pharmacy and NHS stop smoking services in the North of England. Community Dent Oral Epidemiol 2016;44:119-27.

30 Song $F$, Maskrey $\mathrm{V}$, Blyth $\mathrm{A}$, et al. Differences in longer-term smoking abstinence after treatment by specialist or Nonspecialist advisors: secondary analysis of data from a relapse prevention trial. Nicotine Tob Res 2016;18:1061-6.

31 Hackshaw L, McEwen A, West R, et al. Quit attempts in response to smoke-free legislation in England. Tobacco Control 2010;19:160-4.

32 West R. The very best support for stopping smoking. In: UKNSCC-UK Nicotine \& Smoking Cessation Conference London, 2016.

33 Hunt D, Knuchel-Takano A, Jaccard A, et al. Modelling the implications of reducing smoking prevalence: the public health and economic benefits of achieving a 'tobaccofree' UK. Tob Control 2018;27:129-35.

34 Godfrey C, Parrott S, Coleman T, et al. The cost-effectiveness of the English smoking treatment services: evidence from practice. Addiction 2005;100(Suppl 2):70-83. 